\title{
Conducta suicida y trastornos psiquiátricos en Chile, un estudio poblacional
}

\author{
DANIEL SILVA ${ }^{1, a}$, BENJAMÍN VICENTE ${ }^{1}$, \\ SANDRA SALDIVIA ${ }^{1}$, ROBERT KOHN ${ }^{2}$
}

\section{Suicidal behavior and psychiatric disorders in Chile. A population-based study}

Background: Suicidal behavior ranges from ideation to consummation of suicide. In Chile, rates of suicide increased from 4.8 to 12.7/100,000 in the period 1992-2009. Aim: To evaluate the prevalence of suicidal behavior and its relationship with sociodemographic factors and psychiatric diseases. Material and Methods: The Composite International Diagnostic Interview (CIDI), was applied to a representative sample of 2,978 Chilean participants. The prevalence of four suicidal behaviors (thinking about or wishing death, suicide ideation and suicide attempts) and of psychiatric diseases according to the revised third version of the Diagnostic and Statistical Manual of Mental Disorders (DSM III-R), was calculated. Results: The lifetime prevalence of suicidal conception was $14.3 \%$ and the suicide attempt $7.7 \%$, the latest associated with sex, age, school years, smoking habits, being married or having a relationship, depressive disorders, dysthymia, and alcohol, drug and tobacco dependence. Conclusions: Rates founded exceed international prevalence data and extrapolated to current rates of completed suicide, higher levels of suicidal behavior should be expected. Considering the associated disorders we can infer that it is essential a correct diagnosis and treatment of mood disorders and substance consumption to any other specific interventions.

(Rev Med Chile 2013; 141: 1275-1282).

Key words: Mental disorders; Suicidal ideation; suicide.

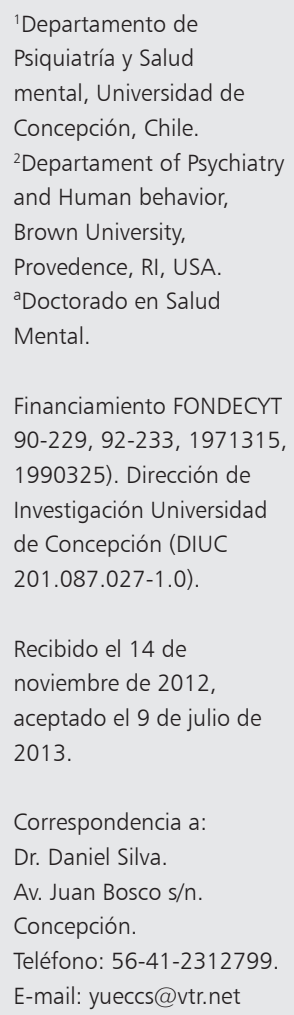

L a conducta suicida puede ser definida como una preocupación o acto que intencionalmente inflija un daño o cause la muerte de si mismo $^{1-3}$. La conducta suicida comprendida como un continuo que abarca desde la ideación hasta el suicidio consumado pasando por el intento de suicido constituye una importante preocupación de salud a nivel mundial tanto en población adulta como adolescente ${ }^{4}$. Las lesiones infligidas a si mismo fueron responsables de 1,3\% de todos los años de vida ajustados por discapacidad de acuerdo al estudio de carga global por enfermedad del año $2000^{5}$. Esto representa una pérdida de años productivos de vida en los jóvenes afectando masivamente a la sociedad según la $\mathrm{OMS}^{6}$.
En el mundo, el suicidio consumado constituye la decimotercera causa de muerte ${ }^{7}$ y las tasas de mortalidad en población general varían de país a país alcanzando cifras muy altas en los países de la Europa del Este como Lituania con 51,6/100.000. En Chile, según los datos del Ministerio de Salud, el suicidio consumado presenta un aumento en las últimas dos décadas con una tasa de 4,8/100.000 en el año 1992 hasta 12,7/100.000 en el 2009, es decir, prácticamente se han triplicado las muertes por esta causa en dicho período, fenómeno que también ha sucedido en otros países latinoamericanos $^{8}$.

Algunos datos indican que la disminución de las tasas de suicidio se relacionaría con el 
Suicidalidad y trastornos psiquiátricos - D. Silva et al

tratamiento de la depresión: prescripciones de antidepresivos y planes para capacitar a médicos en el diagnóstico y tratamiento de la depresión. La caída en las tasas de suicidio en Noruega estuvo relacionada con el aumento de las ventas de antidepresivos no tricíclicos. En Japón 57\% de aumento de prescripciones de antidepresivos se correlaciona con menores tasas de suicidio en el período 1999. 2003, sobre todo en varones. En Estados Unidos de Norteamérica aparece una correlación positiva entre uso de antidepresivos con menor tasa suicida. Varios estudios informan de la disminución de las tasas de suicidio con programas de educación a médicos generales ${ }^{9}$.

La conducta suicida no letal es un factor de riesgo potente para el suicidio y para futuros intentos de suicidio ${ }^{10}$. En relación a la ideación e intento, muy pocos países tienen datos fidedignos ${ }^{7}$. Estudios poblacionales de ideación suicida arrojan tasas de vida variables, altas como $15,7 \%$ en Nueva Zelan$\operatorname{dia}^{11}, 14,8 \%$ en Inglaterra ${ }^{12}$ y $10,9 \%$ en Japón ${ }^{13}$, bajas como en Italia con $3 \%{ }^{14}$. El mismo fenómeno ocurre con el intento de suicidio pero en menor proporción presentando tasas en los estudios poblacionales de 4,5\% en Nueva Zelandia, 4,4\% en Inglaterra, 1,9\% en Japón y 0,5\% en Italia ${ }^{11-14}$.

En Chile los estudios están focalizados en población adolescente escolarizada. Referente a la ideación suicida las cifras son comparativamente mayores a los países previamente señalados, oscilando de $45 \%$ a $57,6 \%{ }^{15-18}$; por otro lado, las prevalencias de vida del intento de suicidio son muy superiores a las internacionales con datos de $14,2 \%$ a $19 \%{ }^{15-18}$.

Si bien es cierto, no todos los actos de autoagresión buscan necesariamente la muerte, hay una fuerte asociación entre infligirse daño a si mismo, intentos de suicidio y suicidio consuma$\mathrm{do}^{19}$. Se sabe que los intentos de suicidio tienden a repetirse y esas repeticiones incrementan el riesgo de un suicidio consumado ${ }^{14}$. En general se acepta que los intentos son 10 a 50 veces más numerosos que los suicidios ${ }^{20}$.

Considerando estos aspectos, conocer la prevalencia de las conductas suicidas y su ideación en la población general es fundamental para visualizar la magnitud del fenómeno en nuestro país. La finalidad de este estudio es estimar la prevalencia de los fenómenos relacionados al suicidio y examinar su asociación con factores sociodemográficos y la comorbilidad psiquiátrica.

\section{Material y Método}

El Estudio Chileno de Prevalencia Psiquiátrica (ECPP) es un estudio basado en una muestra aleatoria estratificada en 3 etapas que consideró comunas, viviendas y posteriormente personas de 15 años o más. El estudio fue diseñado para representar a la población adulta del país. Cuatro provincias fueron elegidas: Santiago, Concepción, Iquique y Cautín, cada una perteneciente a una región diferente del país y que se estimaron representativa de la población nacional. El estudio fue conducido por el Departamento de Psiquiatría y Salud Mental de la Universidad de Concepción entre julio de 1992 y junio de $1999^{21}$.

Los diagnósticos psiquiátricos fueron basados en la DSM-III-R. La entrevista diagnóstica usada para generar estos diagnósticos fue la Composite International Diagnostic Interview (CIDI) (22), versión 1.0 y 1.1. Los 64 entrevistadores que participaron en las distintas etapas del estudio fueron estudiantes universitarios en los últimos años de carreras del área de las ciencias sociales. No se incluyeron estudiantes de medicina o médicos debido a que los entrevistados podrían malinterpretar preguntas acerca de la última vez que habían consultado a un profesional de la salud. La Universidad de Concepción es un centro de referencia y entrenamiento CIDI de la OMS desde 1992, lo que además del exigente protocolo OMS seguido en el entrenamiento de los entrevistadores (80 h de instrucción teórica y práctica) garantiza la comparabilidad de los datos obtenidos en momentos distintos y por entrevistadores nuevos en cada región.

La tasa de respuesta fue $90,3 \%$, lo que determinó un total de 2.987 individuos que finalmente participaron en el estudio; a todos ellos se les aplicó la entrevista CIDI versión 1.0 y 1.1 que incluye 4 preguntas sobre conducta suicida " $¿ A l g u n a$ vez ha tenido un período de dos semanas o más, en que pensó mucho en la muerte?”, “¿Alguna vez ha tenido un período de dos semanas o más en que sintió que quería morirse?”, “¿Alguna vez se ha sentido tan decaído que pensó en suicidarse" y "¿Alguna vez ha intentado suicidarse", además dos secciones del DIS. La tasa de respuesta fue diferente según las provincias $\left(\chi^{2}=11,08 ; \mathrm{df}=3 ; \mathrm{p}<0,02\right)$. Santiago tiene la tasa de no respuesta más alta, $12,6 \%$ e Iquique la más baja, 7,5\%. Los resultados globales del estudio han sido publicados ${ }^{21}$. 
Los sujetos de una población en estudio tienen una probabilidad distinta de ser incluidos en la muestra, esa probabilidad se puede determinar comparando la estructura de la población y de la muestra final. Ese factor o peso corrige las eventuales sobrerrepresentaciones de un estrato o características sociodemográficas determinadas. En consecuencia se determinó un peso para cuantificar la probabilidad de ser seleccionado y participar en el estudio, ello fue considerado al momento de utilizar las pruebas estadísticas.

El presente estudio fue evaluado y aprobado por el comité de ética de la Facultad de Medicina de la Universidad de Concepción y por el comité de ética de la Dirección de Investigación de la misma entidad.

El análisis estadístico se realizó con el programa SPSS 17.0 y SUDAAN, utilizándose estadísticos descriptivos, OR y regresión logística binaria para buscar un modelo multivariado en cada una de las conductas suicidas. Todos los datos son presentados considerando el procedimiento de pesaje.

\section{Resultados}

\section{Prevalencia de vida}

La prevalencia de vida de pensar frecuentemente en la muerte fue de $35,3 \%$, de deseos de estar muerto fue $20,2 \%$, de ideación suicida fue $14,3 \%$ $\mathrm{y}$ de intento de suicidio fue $7,7 \%$.

\section{Factores sociodemogáficos}

En la Tabla 1 se muestra la distribución de la conducta suicida según factores sociodemográficos. Todas las conductas suicidas fueron más frecuentes en mujeres, incluso doblando el número de hombres en el deseo de estar muerto, la ideación suicida y el intento de suicidio. Respecto a la edad, se observa una cierta disminución en las edades mayores respecto a los jóvenes, especialmente en las 2 conductas suicidas más severas. La condición de no conviviente (soltero, separado o viudo) tiene menor prevalencia de intento de suicidio. El nivel educacional presenta una clara relación inversa con las conductas suicidas, mostrando que a mayor

Tabla 1. Distribución de la conducta suicida según factores sociodemográficos

\begin{tabular}{|c|c|c|c|c|c|c|c|c|c|}
\hline & & \multicolumn{2}{|c|}{$\begin{array}{c}\text { Pensar en la } \\
\text { muerte }\end{array}$} & \multicolumn{2}{|c|}{$\begin{array}{l}\text { Deseos de } \\
\text { estar muerto }\end{array}$} & \multicolumn{2}{|c|}{$\begin{array}{l}\text { Ideación } \\
\text { suicida }\end{array}$} & \multicolumn{2}{|c|}{$\begin{array}{l}\text { Intento } \\
\text { suicida }\end{array}$} \\
\hline & & $\mathbf{n}$ & $\%$ & n & $\%$ & $\mathbf{n}$ & $\%$ & n & $\%$ \\
\hline \multirow[t]{2}{*}{ Sexo } & Masculino & 347 & 25,8 & 170 & 11,8 & 138 & 11,4 & 59 & 3,2 \\
\hline & Femenino & 684 & 41,9 & 421 & 27,6 & 281 & 20,7 & 116 & 11,4 \\
\hline \multirow[t]{6}{*}{ Grupos etarios } & $15-24$ & 210 & 34 & 112 & 16,7 & 92 & 15,2 & 41 & 5,2 \\
\hline & $25-34$ & 236 & 31,9 & 140 & 19,1 & 114 & 17,3 & 63 & 9,7 \\
\hline & $35-44$ & 212 & 36 & 123 & 22,4 & 86 & 17,3 & 49 & 6,8 \\
\hline & $45-54$ & 142 & 34,4 & 86 & 22,5 & 62 & 16,4 & 37 & 11,5 \\
\hline & $55-64$ & 110 & 32,3 & 71 & 22,2 & 38 & 17,4 & 25 & 8,3 \\
\hline & $>65$ & 121 & 38,9 & 59 & 21,4 & 27 & 12,2 & 10 & 2,3 \\
\hline \multirow[t]{2}{*}{ Estado de convivencia } & Conviviendo & 496 & 35,7 & 300 & 19,4 & 207 & 16,2 & 129 & 9,1 \\
\hline & No conviviendo & 535 & 33,0 & 291 & 20,9 & 212 & 16,2 & 96 & 5.3 \\
\hline \multirow[t]{4}{*}{ Nivel educacional } & Sin educación & 23 & 40,7 & 13 & 25,9 & 10 & 14,7 & 4 & 5,1 \\
\hline & Básica & 209 & 40,5 & 127 & 24,8 & 82 & 19,3 & 48 & 9,2 \\
\hline & Media & 508 & 37,2 & 307 & 23,1 & 226 & 19,6 & 125 & 9,6 \\
\hline & Superior & 291 & 25,3 & 144 & 12,1 & 101 & 9,0 & 48 & 3,1 \\
\hline \multirow[t]{4}{*}{ Ingresos (UFs) } & $3,17-12,7$ & 556 & 35,0 & 325 & 21,1 & 219 & 14,9 & 123 & 7,1 \\
\hline & $12,71-25,41$ & 186 & 34,8 & 107 & 21,5 & 75 & 17,0 & 38 & 5,9 \\
\hline & $25,42-47,64$ & 101 & 27,7 & 52 & 9,4 & 43 & 13,7 & 14 & 3,8 \\
\hline & $>47,64$ & 76 & 23,6 & 33 & 9,8 & 25 & 11,5 & 14 & 6,3 \\
\hline Hábito tabáquico & Sí & 456 & 37,5 & 281 & 22,7 & 219 & 19,8 & 124 & 10,7 \\
\hline
\end{tabular}


Tabla 2. Riesgos de desarrollar conducta suicida según factores sociodemográficos

\begin{tabular}{|c|c|c|c|c|c|c|c|c|c|}
\hline & & \multicolumn{2}{|c|}{$\begin{array}{c}\text { Pensar en la } \\
\text { muerte }\end{array}$} & \multicolumn{2}{|c|}{$\begin{array}{l}\text { Deseos de estar } \\
\text { muerto }\end{array}$} & \multicolumn{2}{|c|}{ Ideación suicida } & \multicolumn{2}{|c|}{$\begin{array}{l}\text { Intento de } \\
\text { suicidio }\end{array}$} \\
\hline & & OR & IC $95 \%$ & OR & IC $95 \%$ & OR & IC $95 \%$ & OR & IC $95 \%$ \\
\hline \multirow[t]{2}{*}{ Sexo } & Masculino & 1 & & 1 & & 1 & & 1 & \\
\hline & Femenino & 2,07 & $(1,59-2,69)$ & 2,86 & $(2,25-3,63)$ & 2,03 & $(1,39-2,97)$ & 3,90 & $(2,36-6,43)$ \\
\hline \multirow{6}{*}{$\begin{array}{l}\text { Grupos } \\
\text { etarios }\end{array}$} & $15-24$ & 0,81 & $(0,53-1,25)$ & 0,73 & $(0,36-1,49)$ & 1,29 & $(0,61-2,75)$ & 2,37 & $(0,86-6,53)$ \\
\hline & $25-34$ & 0,73 & $(0,47-1,14)$ & 0,87 & $(0,46-1,65)$ & 1,51 & $(0,85-2,69)$ & 4,68 & $(1,81-12,13)$ \\
\hline & $35-44$ & 0,88 & $(0,59-1,32)$ & 1,06 & $(0,54-2,06)$ & 1,51 & $(0,65-3,50)$ & 3,18 & $(1,32-7,64)$ \\
\hline & $45-54$ & 0,82 & $(0,47-1,44)$ & 1,06 & $(0,63-1,81)$ & 1,41 & $(0,73-2,72)$ & 5,61 & $(2,00-15,77)$ \\
\hline & $55-64$ & 0,75 & $(0,41-1,37)$ & 1,05 & $(0,53-2,05)$ & 1,52 & $(0,61-3,82)$ & 3,92 & $(1,42-10,80)$ \\
\hline & $>65$ & 1,0 & & 1,0 & & 1,0 & & 1,0 & \\
\hline \multirow[t]{2}{*}{ Estado civil } & Conviviendo & 0,89 & $(0,70-1,12)$ & 0,91 & $(0,67-1,24)$ & 1,01 & $(0,82-1,23)$ & 1.08 & $(1,06-3,06)$ \\
\hline & No conviviendo & 1,0 & & 1,0 & & 1,0 & & 1,0 & \\
\hline \multirow{4}{*}{$\begin{array}{l}\text { Nivel } \\
\text { educacional }\end{array}$} & Sin educación & 2,03 & $(0,80-51,9)$ & 2,55 & $(0,67-9,71)$ & 1,73 & $(0,41-7,27)$ & 1,68 & $(0,41-6,85)$ \\
\hline & Básica & 2,01 & $(1,29-3,13)$ & 2,40 & $(1,46-3,96)$ & 2,40 & $(1,30-4,43)$ & 3,15 & $(1,47-6,77)$ \\
\hline & Media & 1,75 & $(1,42-2,17)$ & 2,19 & $(1,56-3,07)$ & 2,45 & $(1,59-3,75)$ & 3,31 & $(1,83-5,99)$ \\
\hline & Superior & 1,0 & & 1,0 & & 1,0 & & 1,0 & \\
\hline \multirow{4}{*}{$\begin{array}{l}\text { Ingresos } \\
\text { (UFs) }\end{array}$} & $3,17-12,7$ & 1,74 & $(1,21-2,50)$ & 2,47 & $(1,84-3,31)$ & 1,35 & $(0,66-2,79)$ & 1,14 & $(0,47-2,77)$ \\
\hline & $12,71-25,41$ & 1,73 & $(1,14-2,62)$ & 2,52 & $(1,78-3,57)$ & 1,58 & $(0,83-3,01)$ & 0,94 & $(0,26-3,42)$ \\
\hline & $25,42-47,64$ & 1,24 & $(0,96-1,60)$ & 0,96 & $(0,53-1,73)$ & 1,23 & $(0,65-2,32)$ & 0,60 & $(0,33-1,09)$ \\
\hline & $>47,64$ & 1,0 & & 1,0 & & 1,0 & & 1,0 & \\
\hline \multirow{2}{*}{$\begin{array}{l}\text { Hábito } \\
\text { tabáquico }\end{array}$} & Sí & 1,30 & $(1,04-1,62)$ & 1,35 & $(1,09-1,67)$ & 1,58 & $(1,19-2,09)$ & 2,28 & $(1,46-3,57)$ \\
\hline & No & 1 & & 1 & & 1 & & 1 & \\
\hline
\end{tabular}

número de años de estudio son menos frecuentes; de manera muy similar a lo observado con los ingresos económicos de la familia.

\section{Riesgo de desarrollar conducta suicida}

En la Tabla 2 se presentan los riesgos de desarrollar conductas suicidas según factores sociodemográficos. Es claro que las mujeres doblan el riesgo de los hombres e incluso lo triplican en el intento de suicidio. Observamos que en la conducta suicida más severa, los adultos mayores están menos expuestos a estas conductas comparándolas con los jóvenes, tendencia que si bien no es significativa, se repite en la ideación y el deseo de estar muerto. El estado de convivencia presenta una mayor presencia de intentos de suicidio de manera estadísticamente significativa mientras que a menor nivel educacional existe mayor riesgo de cualquiera de las conductas suicidas, excepto con los individuos sin educación. Al observar los ingresos familiares, vemos que quienes tienen ingresos más bajos presentan más riesgo para las conductas suicidas menos severas. La presencia del hábito tabáquico se asoció de manera estadísticamente significativas con la presencia de todas las conductas suicidas.

$\mathrm{Al}$ ingresar las variables sociodemográficas a la regresión logística binaria teniendo como variable respuesta cada una de las 4 conductas suicidas observamos que para "Pensar frecuentemente en la muerte" ingresaron en el modelo significativamente el sexo $(\mathrm{p}<0,001)$, la edad entre 35 y 44 años $(p=0,03)$, el estado de convivencia $(\mathrm{p}<0,001)$ y los años de escolaridad $(\mathrm{p}=0,001)$; para los "Deseos de estar muerto" fueron significativos el sexo ( $p<0,001)$, los años de escolaridad ( $p=0,003)$, el ingreso económico familiar $(\mathrm{p}>0,019)$, el hábito tabáquico $(\mathrm{p}=0,001)$ y el estado de convivencia $(\mathrm{p}=0,003)$. Para la ideación suicida ingresaron en el modelo de manera significativa el sexo $(p=0,004)$, la edad $(p=0,003)$, el hábito tabáquico $(\mathrm{p}<0,001)$, la condición de 
Suicidalidad y trastornos psiquiátricos - D. Silva et al

Tabla 3. Riesgo de conducta suicida para cada diagnóstico DSM-III-R a lo largo de la vida en el análisis multivariado de la regresión logística binaria

\begin{tabular}{|c|c|c|c|c|c|c|c|c|}
\hline & \multicolumn{2}{|c|}{$\begin{array}{c}\text { Pensar en la } \\
\text { muerte }\end{array}$} & \multicolumn{2}{|c|}{$\begin{array}{c}\text { Deseos de estar } \\
\text { muerto }\end{array}$} & \multicolumn{2}{|c|}{$\begin{array}{l}\text { Ideación } \\
\text { suicida }\end{array}$} & \multicolumn{2}{|c|}{$\begin{array}{l}\text { Intento de } \\
\text { suicidio }\end{array}$} \\
\hline & $\mathbf{p}$ & OR & $\mathbf{p}$ & OR & $\mathbf{p}$ & OR & $\mathbf{p}$ & OR \\
\hline Trastorno cognitivo & 0,111 & 1,635 & 0,699 & 1,163 & 0,895 & 1,059 & 0,344 & 1,574 \\
\hline Trastorno psicótico & 0,001 & 3,323 & 0,001 & 3,005 & 0,000 & 3,763 & 0,095 & 1,906 \\
\hline Bipolaridad & 0,000 & 4,878 & 0,000 & 4,279 & 0,004 & 2,796 & 0,312 & 1,581 \\
\hline Trastorno depresivo & 0,000 & 5,974 & 0,000 & 5,361 & 0,000 & 4,817 & 0,000 & 2,734 \\
\hline Distimia & 0,000 & 1,986 & 0,000 & 2,949 & 0,000 & 2,444 & 0,000 & 2,927 \\
\hline Trastorno de pánico & 0,057 & 2,338 & 0,393 & 1,413 & 0,797 & 0,899 & 0,538 & 1,325 \\
\hline Agorafobia & 0,808 & 1,108 & 0,011 & 3,617 & 0,032 & 3,042 & 0,120 & 2,677 \\
\hline Ansiedad generalizada & 0,006 & 2,420 & 0,003 & 2,410 & 0,060 & 1,747 & 0,055 & 1,844 \\
\hline Cualquier fobia & 0,115 & 1,872 & 0,306 & 0,606 & 0,525 & 0,728 & 0,548 & 0,692 \\
\hline Abuso alcohol & 0,201 & 1,387 & 0,575 & 0,836 & 0,224 & 1,469 & 0,205 & 0,507 \\
\hline Dependencia alcohol & 0,105 & 1,411 & 0,457 & 1,193 & 0,003 & 2,023 & 0,026 & 1,827 \\
\hline Abuso drogas & 0,564 & 1,321 & 0,312 & 1,689 & 0,006 & 3,772 & 0,244 & 1,889 \\
\hline Dependencia drogas & 0,008 & 2,547 & 0,000 & 4,020 & 0,004 & 2,658 & 0,000 & 3,685 \\
\hline Dependencia tabaco & 0,084 & 1,687 & 0,010 & 2,217 & 0,001 & 2,727 & 0,037 & 2,104 \\
\hline Trastorno somatización & 0,601 & 0,865 & 0,725 & 0,903 & 0,032 & 1,822 & 0,728 & 1,121 \\
\hline Personalidad antisocial & 0,518 & 1,299 & 0,117 & 1,880 & 0,191 & 1,727 & 0,077 & 2,197 \\
\hline
\end{tabular}

Tabla 4. Riesgo de conducta suicida según número de comorbilidades psiquiátricas, de acuerdo a diagnóstico DSM-III-R

\begin{tabular}{|lcccccccc|}
\hline & \multicolumn{2}{c}{$\begin{array}{c}\text { Pensar en la } \\
\text { muerte }\end{array}$} & \multicolumn{2}{c}{$\begin{array}{c}\text { Deseos de estar } \\
\text { muerto }\end{array}$} & \multicolumn{2}{c|}{ Ideación suicida } & \multicolumn{2}{c|}{ Intento suicida } \\
Comorbilidad & OR & IC 95\% & OR & IC 95\% & OR & IC 95\% & OR & IC 95\% \\
\hline Ninguna & 1 & & 1 & & 1 & & 1 & \\
\hline 1 & 3,10 & $(2,30-4,18)$ & 4,00 & $(2,35-6,79)$ & 3,14 & $(1,90-5,20)$ & 4,13 & $(2,11-8,08)$ \\
\hline 2 & 5,40 & $(4,08-7,15)$ & 8,83 & $(5,52-14,12)$ & 9,47 & $(4,85-18,51)$ & 9,33 & $(4,04-21,56)$ \\
\hline 3 o más & 12,43 & $(7,05-21,90)$ & 18,61 & $(8,96-38,64)$ & 15,25 & $(7,62-30,54)$ & 15,23 & $(8,89-26,11)$ \\
\hline
\end{tabular}

convivencia $(p=0,021)$ y los años de estudio $(\mathrm{p}<0,001)$. Finalmente, para el intento de suicidio fueron significativos el sexo $(\mathrm{p}<0,001)$, la edad $(\mathrm{p}=0,019)$, los años de escolaridad $(\mathrm{p}=0,002)$ y el hábito tabáquico $(\mathrm{p}=0,004)$.

\section{Comorbilidad psiquiátrica y conducta suicida}

En la Tabla 3 se muestran los trastornos psiquiátricos como factores asociados a la conducta suicida en el análisis multivariado de la regresión logística binaria junto a sus OR (IC 95\%). Para "Pensar frecuentemente en la muerte" resultaron significativos los trastornos depresivos, psicótico, distimia, bipolaridad, ansiedad generalizada y de- pendencia a drogas. Para "Deseos de estar muerto" se incluyeron los trastornos depresivos, psicótico, distimia, bipolaridad, ansiedad generalizada, agorafobia y dependencia a drogas y tabaco. Para la ideación suicida fueron significativos el trastorno psicótico, bipolaridad, trastorno depresivo, distimia, agorafobia, dependencia a alcohol, abuso y dependencia a drogas, dependencia de tabaco y trastorno por somatización. Finalmente, para el intento de suicidio fueron estadísticamente significativos el trastorno depresivo, distimia y dependencia de alcohol, drogas y tabaco.

En la Tabla 4 se presenta el riesgo de conducta suicida y el número de cuadros comórbidos según 
DSM-III-R, apreciándose que sin importar el tipo de patología, todas ellas llevan a un aumento significativo del riesgo de suicidalidad.

\section{Discusión}

Los resultados muestran una prevalencia elevada de la conducta suicida en la población chilena, por sobre Nueva Zelanda y una proporción de intento de suicidio muy superior a Japón, país donde este fenómeno es reconocidamente relevante. Los hallazgos son aún más llamativos si se considera que son datos recogidos durante la década pasada, y que, como ya se mencionó, el Ministerio de Salud ha reportado tasas de suicidio consumado en aumento desde 1992, las que prácticamente se triplicaron entre ese año y el 2009. Si se tiene en consideración la teoría del continuo de la suicidalidad, es posible asumir que actualmente las tasas de ideación y de intento que no consultan a los dispositivos de atención médica y psicológica son mucho mayores a las aquí reportadas, lo que abre la interrogante acerca de si el sistema sanitario puede dar acogida de manera óptima a todos esos casos, lo que requiere que los procedimientos de búsqueda dirigida para la detección de conductas suicidas no letales sea realizada de manera rutinaria en la atención primaria.

Las tasas encontradas en la población general son menores a las reportadas en estudios sobre muestras de adolescentes chilenos, apoyando las teorías que la suicidalidad va en disminución con la edad y coloca el acento en la necesidad de contar con estrategias específicas dirigidas a esta población.

Concordante con los estudios internacionales, el sexo femenino, los jóvenes, la condición de conviviente y las desventajas sociales (años de estudio e ingresos familiares) resultan marcadamente asociados a una mayor prevalencia de conducta suicida. Un hallazgo aparte es el consumo de tabaco que ingresa de manera independiente en el modelo multivariado tanto para la ideación suicida como para el intento de suicidio, pese a que no hay un modelo teórico explicativo claro para ello, aparentemente se asociaría a individuos más impulsivos que tienden al uso de sustancias ${ }^{23}$.

Los hallazgos referentes a la comorbilidad asociada son consistentes con lo reportado en otros estudios, es decir, los trastornos psicóticos, los trastornos del ánimo y el Abuso/Dependencia de sustancias se asocian a una mayor frecuencia de conducta suicida; pero hay escasa evidencia de la relación entre trastornos ansiosos y conducta suicidas. Es posible que este hallazgo este asociado a una eventual discusión respecto de la vinculación entre trastornos depresivos y ansiosos y que estos últimos tengan menor peso en el modelo multivariado, por lo que no aparecen como significativamente relacionados. Nuevamente esta evidencia nos lleva a pensar que el primer paso para reducir la suicidalidad en la población es una mejor pesquisa y tratamiento de los trastornos del ánimo, por ello evaluar el impacto del programa GES de depresión, relativo a la disminución de la conducta suicida es una tarea pendiente.

Finalmente, los hallazgos se contraponen a uno de los argumentos más frecuentes en relación a la no instauración de medidas concretas para prevenir la suicidalidad, y es que éste sería un fenómeno poco frecuente en la actualidad, lo que queda totalmente desmentido.

\section{Limitaciones}

La principal limitación de este estudio, como ya lo hemos mencionado es la antigüedad de sus datos que reflejan la situación de la población nacional de los años '90. Pese a ello, este es el primer y único estudio poblacional chileno relativo a la conducta suicida por lo que aporta información que no poseíamos y que es un punto de partida para nuevos avances. Debemos considerar que, si bien los datos del ECPP tienen ya cerca de 20 años, su información aún es utilizada para la estructuración de planes en salud mental en el país por lo que las nuevas estrategias contra la suicidalidad podrán contar desde ahora con datos concretos para su planes estratégicos. Además, a la luz de datos obtenido de un estudio longitudinal en población consultante en centros de atención primaria de salud de la provincia de Concepción tomados el año 2006, que refleja tasas de $20,8 \%$ para la ideación suicida y de 9,7\% de intento de suicidio (Vicente B, Kohn R, Saldivia S. The prevalence of psychiatric disorders in primary care in Chile. Libro de resúmenes del $162^{\text {nd }}$ Annual Meeting American Psychiatric Association. San Francisco. 2009: 278) vemos que las tasas en población nacional son levemente menores lo que es explicado por la condición de consultantes de la muestra referenciada y a su vez revela que los niveles de 
suicidalidad presentan cierta tendencia a la estabilización. Otra limitación es que los datos fueron reportados de manera retrospectiva y pueden estar bajo el sesgo del recuerdo ya que no se confrontó la información con la aportada por terceros; pese a ello existen revisiones sistemáticas que avalan esta metodología ya que los adultos pueden recordar experiencias pasadas con la suficiente precisión para proveer información valiosa ${ }^{24}$.

\section{Referencias}

1. Pfeffer, C. Suicidal Behavior in Children and Adolescents: causes and management. En Lewis, Melvin (Ed) Child and Adolescent Psychiatry a Comprehensive Text Book. 2007. 4th Edition Williams and Wilkins, Pgs 52938.

2. Fundutis T. Mood disorders, suicide and suicide behavior. Curr Opin Pediatr 1990; 2: 700-4.

3. Arias M, Marcos S, Martín M, Arias J, Deronceré O. Modificaciones de conocimientos sobre conducta suicida en adolescentes y adultos jóvenes con riesgo. MEDISAN 2009. 13 (1).

4. Pfeffer C, Martins P, Mann J, Sunkenberg M, Ice A, Damore J, et al. Child Survivors of Suicide: Psychosocial Characteristics. Journal of the American Academy of Child \& Adolescent Psychiatry 1997; 36 (1): 65-74.

5. Ustun TB, Ayuso-Mateos JL, Chatterji S, Mathers C, Murray JL. Global burden of depressive disorders in the year 2000. Br J Psychiatry 2004; 184: 386-92.

6. Porter D, Bertolote J. El suicidio, un problema de salud pública enorme y sin embargo prevenible, según la OMS. OMS 2004. http://www.who.int/mediacentre/ news/releases/2004/pr61/es/index.html [Consultado por última vez el 6/10/12].

7. Krug E, Mercy J, Dahleberg L, Zwi A, The World Report on Violence and Health. The Lancet 2002; 360 (9339): 1083-8.

8. Kohn R, Friedmann H. La epidemiología del suicidio los intentos y las ideaciones suicidas en América Latina y el Caribe. En: Rodríguez J, Kohn R, Aguilar-Gaxiola S. Epidemiología de los trastornos mentales en América Latina y el Caribe 2009. Organización Panamericana de la Salud. p. 193-207.

9. Retamal, P. Suicidio: Problemas y prevención. Boletín informativo de Salud Mental 2010; 4: 3-7.

10. Beautrais AL, Collings SCD, Ehrhardt P, Henare K. Suicide prevention: a review of evidence of risk and protective factors, and points of effective intervention. Wellington: Ministry of Health, 2005: 82.

11. Beautrais A, Wells E, McGee M, Oakley M. Suicidal be- haviour in Te Rau Hinengaro: The New Zealand Mental Health Survey. Australian and New Zealand Journal of Psychiatry 2006: 40; 896-904.

12. Bebbington P, Minot S, Cooper C, Dennis M, Meltzer $\mathrm{H}$, Jenkins $\mathrm{R}$, et al. Suicidal ideation, self-harm and attempted suicide: Results from the British psychiatric morbidity survey 2000. European Psychiatry 2010; 25: 427-31.

13. Ono Y, Kawakami N, Nakane Y, Nakamura Y, Tachimori $\mathrm{H}$, Iwata N, et al. Prevalence of and risk factors for suicide-related outcomes in the World Health Organization World Mental Health Surveys Japan. Psychiatry and clinical Neurosciences 2008; 62: 442-9.

14. Scocco P, de Girolamo G, Vitagut G, Alonso J. Prevalence of suicide ideation, plans and attempts and related risk factors in Italy: Results from the European Study on the Epidemiology of Mental Disorders-World Mental Health study. Comprehensive Psychiatry 2008; 49: 13-21.

15. Salvo L, Rioseco P, Salvo S. Ideación suicida e intento de suicidio en adolescentes de enseñanza media. Rev Chil Neuropsiquiat 1998; 36: 28-34.

16. Ventura-Juncá R, Carvajal C, Undurraga S, Vicuña P, Egaña J, Garib M. Prevalencia de la ideación e intento suicida en adolescentes de la Región Metropolitana de Santiago de Chile. Rev Med Chile 2010; 138: 309-15.

17. Valdivia M, Quevedo I, Silva D, Salazar P, Torres S. Factores de riesgo asociados a intento de suicidio adolescente en una comuna rural de la provincia de Concepción. Revista Sociedad de Psiquiatría y Neurología de la Infancia y Adolescencia. Libro de resúmenes congreso 2004. 2005; 16 (1): 60.

18. Haquin C, Larraguibel M, Cabezas J. Factores protectores y de riesgo en salud mental en niños y adolescentes de la ciudad de Calama. Rev Chil Pediatr 2004; 75 (5): 425-33.

19. Owens D, Wood C, Greenwood D, Hughes T, Dennis M. Mortality and suicide after non-fatal poisoning: a 16-year outcome study of patients attending accident an emergency. Br J Psychiatry 2005; 187: 470-5.

20. Casey P, Dunn G, Kelly B, Lehtinen V, Dalgard O, Dowrick $\mathrm{C}$, et al. The prevalence of suicidal ideation in the general population: results from the Outcome of Depression International Network (ODIN) study. Soc Psychiatry Psychiatr Epidemiol 2008; 43: 299-304.

21. Vicente B, Rioseco P, Saldivia S, Kohn R, Torres S. Estudio chileno de prevalencia de patología psiquiátrica (DSM-III-R/CIDI) (ECPP). Rev Med Chile 2002; 130: 527-36.

22. Robins LN, Wing J, Wittchen HU, Helzer JE, Babor TF, Burke J, et al. The Composite International Diagnostic Interview: an epidemiologic instrument suitable for use 
in conjunction with different diagnostic systems and in different cultures. Arch Gen Psychiatry 1988; 45: 106977.

23. Whelan R, Conrod P, Poline J, Lourdusamy A, Banaschewski T, Barker G, et al. Adolescent impulsivity phe- notypes characterized by distinct brain networks. Nature Neuroscience 2012; 15: 920-5.

24. Hardt J, Rutter M. Validity of adult retrospective reports of adverse childhood experiences: Review of the evidence. J Child Psychol Psychiatry 2004; 45: 260-73. 